Bull. Korean Math. Soc. 50 (2013), No. 5, pp. 1753-1763

http://dx.doi.org/10.4134/BKMS.2013.50.5.1753

\title{
CYCLIC SUBGROUP SEPARABILITY OF CERTAIN GRAPH PRODUCTS OF SUBGROUP SEPARABLE GROUPS
}

\author{
Kok Bin Wong And Peng Choon Wong
}

\begin{abstract}
In this paper, we show that tree products of certain subgroup separable groups amalgamating normal subgroups are cyclic subgroup separable. We then extend this result to certain graph product of certain subgroup separable groups amalgamating normal subgroups, that is we show that if the graph has exactly one cycle and the cycle is of length at least four, then the graph product is cyclic subgroup separable.
\end{abstract}

\section{Introduction}

Cyclic subgroup separability or $\pi_{c}$ was introduced by Stebe [22]. Kim [12, 13] had given useful criteria for certain generalized free products and HNN extensions to be cyclic subgroup separable. By using Kim's criterion for HNN extensions, Wong and Wong [28] had given a characterization for certain HNN extensions with central associated subgroups to be cyclic subgroup separable. Kim and Tang [17] had given a sufficient and necessary condition for HNN extensions of cyclic subgroup separable groups with cyclic associated subgroups to be cyclic subgroup separable.

Cyclic subgroup separability is used to show that certain generalized free products are conjugacy separable (see $[15,16,23,24]$ ). Conjugacy separability is used by Grossman [8] to show that certain outer automorphism groups are residually finite. In fact, she showed that if all the class-preserving automorphisms of a finitely generated conjugacy separable group $G$ are inner, then the outer automorphism group of $G$ is residually finite. This criterion has been used by many authors to show that certain outer automorphism groups are residually finite (see $[2,3,6,14,18,26,29])$. Recently, Zhou and Kim [31, 32] had studied the class-preserving automorphisms of certain groups. Raptis, Talelli and Varsos [21] showed that conjugacy separability and residually finiteness are equivalent in certain HNN extensions (see also $[26,27]$ for similar results).

Received January 10, 2013.

2010 Mathematics Subject Classification. Primary 20E06, 20E26; Secondary 20F10.

Key words and phrases. cyclic subgroup separability, graph products, subgroup separable, polycyclic-by-finite groups. 
Subgroup separability is a property stronger than cyclic subgroup separability. It is well known that polycyclic groups and free groups are subgroup separable (Hall [9], Mal'cev [19]). Since a finite extension of a subgroup separable group is again subgroup separable, polycyclic-by-finite groups and free-by-finite groups are subgroup separable. Metaftsis and Raptis [20] gave a sufficient and necessary condition for certain HNN extensions to be subgroup separable. By applying their result, Wong and Wong [30] showed that subgroup separability and conjugacy separability are equivalent in certain HNN extensions.

In this paper, we will study cyclic subgroup separability of certain graph products. This paper is motivated by the works of Kim [11], Allenby [1], and Wong and Wong [25]. We will give a generalization of the Allenby's Theorem $[1$, Theorem C], which is a generalization of the Kim's Theorem [11, Theorem 2.11]. In Section 2, we will discuss the generalization of Allenby's Theorem (see Theorem 2.5). In Sections 3, 4 and 5, we will provide the details of the proofs.

\section{Generalizing Allenby's theorem}

The notation used here is standard. In addition, the following will be used for any group $G, N \triangleleft_{f} G$ means $N$ is a normal subgroup of finite index in $G$. We denote by $A_{H}^{*} B$ the generalised free product of $A$ and $B$ with the subgroup $H$ amalgamated. If $G=A_{H}^{*} B$ and $x \in G$, then $\|x\|$ denotes the free product length of $x$ in $G$. If $\bar{G}$ is a homomorphic image of $G$, then we use $\bar{x}$ to denote the image of $x$ in $\bar{G}$.

Definition 2.1. A group $G$ is called $H$-separable for the subgroup $H$ if for each $x \in G \backslash H$, there exists $N \triangleleft_{f} G$ such that $x \notin H N$.

$G$ is called $H K$-separable for the subgroups $H, K$ if for each $x \in G \backslash H K$, there exists $N \triangleleft_{f} G$ such that $x \notin H K N$.

A group $G$ is termed subgroup separable if $G$ is $H$-separable for every finitely generated subgroup $H$. A group $G$ is termed cyclic subgroup separable (or $\pi_{c}$ for short) if $G$ is $H$-separable for every cyclic subgroup $H$. A group $G$ is termed residually finite if $G$ is 1-separable.

Definition 2.2. Let $Q$ be a simple graph (without loops and multiple edges) with vertex set $V(Q)$ and edge set $E(Q)$. To each vertex $v$ of $Q$ assign a vertex group $A_{v}$, and to each edge $e$ of $Q$ assign an edge group $H_{e}$ together with monomorphisms $\alpha_{e}$ and $\beta_{e}$ embedding $H_{e}$ into the two vertex groups at the end of $e$. The graph product $G(Q)$ of the vertex groups amalgamating the edge groups is defined to be the group generated by all the generators of the vertex groups with defining relations given by the defining relations of all the vertex groups together with the relations $\alpha_{e}\left(g_{e}\right)=\beta_{e}\left(g_{e}\right)$ for each $g_{e}$ in $H_{e}$.

Roughly speaking, if $i j$ is an edge in $E(Q)$, then $H_{i j}$ is a subgroup of $A_{i}$ and $A_{j}$. Since $i j$ and $j i$ represent the same edge in $Q$, we have $H_{i j}=H_{j i}$.

If $Q$ is a tree, then $G(Q)$ is called the tree product, whereas if $Q$ is a cycle (polygon), then $G(Q)$ is called the polygonal product. 
The polygonal products of groups were introduced by Karrass, Pietrowski and Solitar [10] in their study of the subgroup structure of the Picard group $\operatorname{PSL}(2, \mathbb{Z}[i])$. By using their results, Brunner, Frame, Lee and Wielenberg [5] characterized all the torson-free subgroups of finite index in the Picard group. Polygonal products also form a large subclass in the class of one-relator products of cyclic groups. For certain one-relator products, Fine, Howie and Rosenberger [7] had proved a Freiheitssatz but the word problem and residual finiteness are still unknown.

Definition 2.3. Let $u, v \in V(Q)$. If $u$ is adjacent to $v$ in $Q$, i.e., $u v \in E(Q)$, then we shall write $u \sim v$.

Suppose that for each $u \in V(Q)$, the edge group $H_{u v}$ is normal in the vertex group $A_{u}$ for all $v \in V(Q)$ with $v \sim u$. We say that the edge groups satisfy the intersection property if for each edge $u v \in E(Q)$,

$$
H_{u v} \cap \prod_{\substack{w \sim u, w \neq v}} H_{u w}=1 .
$$

We remark here that, since all the edge groups are normal in its vertex group, the product $\prod_{w \sim u, w \neq v} H_{u w}$ is the subgroup generated by all the edge groups $\left\langle H_{u w}: w \sim u, w \neq v\right\rangle$ in $A_{u}$. In fact, if the edge groups satisfy the intersection property, the subgroup generated by all the edge groups in $A_{u}$ is the direct product

$$
\bigotimes_{w \sim u} H_{u w}
$$

Note that if $Q$ is a cycle of length 4 and the intersection property holds, then we have vertex groups $A_{1}, A_{2}, A_{3}, A_{4}$ and edge groups $H_{1}, H_{2}, H_{3}, H_{4}$ such that $A_{i} \cap A_{i+1}=H_{i}$ and $H_{i} \cap H_{i+1}=1$ for $i=1,2,3,4$ where the subscripts are taken modulo 4. What Allenby [1, Theorem C] has proved is the following theorem (see also [25, Theorem 4.6]):

Theorem 2.4 (Allenby's Theorem). If $Q$ is a cycle of length at least 4 and the intersection property holds, then the polygonal product $G(Q)$ of polycyclicby-finite groups amalgamating finitely generated normal subgroups is $\pi_{c}$.

The objective of this paper is to prove the following theorem, which is a generalization of Theorem 2.4.

Theorem 2.5. Suppose $Q$ is a simple graph that has exactly one cycle. If the length of the cycle is at least 4 and the intersection property holds, then the graph product $G(Q)$ of subgroup separable groups amalgamating finitely generated normal subgroups is $\pi_{c}$.

Since polycyclic-by-finite is subgroup separable, the following corollary is a consequence of Theorem 2.5.

Corollary 2.6. Suppose $Q$ is a simple graph that has exactly one cycle. If the length of the cycle is at least 4 and the intersection property holds, then the 
graph product $G(Q)$ of polycyclic-by-finite groups amalgamating normal subgroups is $\pi_{c}$.

We shall need the following result of Kim [12, Proposition 1.2].

Theorem 2.7. Let $G=A_{H}^{*} B$. Suppose that

(a) $A$ and $B$ are $\pi_{c}$ and $H$-separable,

(b) for each $N \triangleleft_{f} H$, there exist $N_{A} \triangleleft_{f} A$ and $N_{B} \triangleleft_{f} B$ such that $N_{A} \cap H=$ $N_{B} \cap H \subset N$.

Then $G$ is $\pi_{c}$.

Note that to prove Theorem 2.5, we may assume that $Q$ is connected. Let $C$ be the cycle of length at least 4 in $Q$. Then $C$ contains at least 4 vertices, say $u_{1}, u_{2}, v_{1}, v_{2}$, such that $u_{1} \sim v_{1}$ and $u_{2} \sim v_{2}$. Now if we remove the edges $u_{1} v_{1}$ and $u_{2} v_{2}$ from $Q$, then the resulting graph $Q-\left\{u_{1} v_{1}, u_{2} v_{2}\right\}$, is the union of two trees, say $T_{1}$ and $T_{2}$. Since the intersection property holds, we conclude that the subgroup generated by $H_{u_{1} v_{1}}$ and $H_{u_{2} v_{2}}$ in $G\left(T_{1}\right)$ is the free product $H_{u_{1} v_{1}} * H_{u_{2} v_{2}}$. Similarly, the free product $H_{u_{1} v_{1}} * H_{u_{2} v_{2}}$ is the subgroup generated by $H_{u_{1} v_{1}}$ and $H_{u_{2} v_{2}}$ in $G\left(T_{2}\right)$. Therefore

$$
G(Q)=G\left(T_{1}\right)_{\left(H_{u_{1} v_{1} * H_{\left.u_{2} v_{2}\right)}}\right.}^{*} G\left(T_{2}\right) .
$$

If we could show that $G\left(T_{1}\right)$ and $G\left(T_{2}\right)$ satisfy the conditions (a) and (b) of Theorem 2.7, then Theorem 2.5 follows.

From now onwards throughout the paper, we shall assume the following:

(a) $T$ is a tree;

(b) the intersection property holds;

(c) $G(T)$ is the tree product of subgroup separable groups amalgamating finitely generated normal subgroups;

(d) the vertex groups are denoted by $A_{u}, u \in V(T)$, and the edge groups are denoted by $H_{u v}, u v \in E(T)$.

Now, Theorem 2.5 follows from Theorem 2.7 applying Theorem 2.8, Lemma 2.9 and Lemma 2.10 .

Theorem 2.8. $G(T)$ is $\pi_{c}$.

Lemma 2.9. Let $M$ and $K$ be finitely generated normal subgroups of $A_{r}$ and $A_{k}$ respectively where $M \cap \prod_{j \sim r} H_{r j}=1=K \cap \prod_{j \sim k} H_{k j}$ and $r \neq k$. Then $G(T)$ is $M * K$-separable.

Let $G$ be a finitely generated group and $S \triangleleft_{f} G$. If $S$ is a characteristic subgroup of $G$, then we set $f_{G}(S)=S$. Suppose $S$ is not a characteristic subgroup of $G$. Let $[G: S]=m$ where $m$ is a positive integer. Since $G$ is finitely generated, the number of subgroups of index $m$ in $G$ is finite. Let $N$ be the intersection of all these subgroups. Then $N$ is a characteristic subgroup of finite index in $G$ and $N \subseteq S$. We set $f_{G}(S)=N$ (see [25, Lemma 3.1]). 
Lemma 2.10. Let $M$ and $K$ be finitely generated normal subgroups of $A_{r}$ and $A_{k}$ respectively where $M \cap \prod_{j \sim r} H_{r j}=1=K \cap \prod_{j \sim k} H_{k j}$ and $r \neq k$. Then for each $S \triangleleft_{f}(M * K)$, there exists $N \triangleleft_{f} G(T)$ such that $N \cap(M * K)=f_{M * K}(S)$.

\section{Proof of Theorem 2.8}

Lemma 3.1. Let $A$ be a subgroup separable group and $H_{1}, H_{2}, \ldots, H_{n}$ be finitely generated normal subgroups of $A$ such that $H_{i} \cap \prod_{j \neq i} H_{j}=1$ for $i=1,2, \ldots, n$. If $S_{i} \triangleleft_{f} H_{i}$, then there exists $N \triangleleft_{f} A$ such that $N \cap H_{i}=f_{H_{i}}\left(S_{i}\right)$, and $N H_{i} \cap N H_{j}=N, j \neq i, 1 \leq i, j \leq n$.

Proof. Let $S=\Pi_{i=1}^{n} f_{H_{i}}\left(S_{i}\right)$ and $H=\Pi_{i=1}^{n} H_{i}$. Since $H_{i}$ is finitely generated and $f_{H_{i}}\left(S_{i}\right) \triangleleft_{f} H_{i}$, we have $f_{H_{i}}\left(S_{i}\right)$ is finitely generated and thus $S$ is finitely generated. Note that $S$ is a finitely generated normal subgroup in $A$. Therefore $\bar{A}=A / S$ is residually finite and $\bar{H}=H / S$ is finite. So, there exists $\bar{N} \triangleleft_{f} \bar{A}$ such that $\bar{N} \cap \bar{H}=1$. Let $N$ be the preimage of $\bar{N}$. Now, we show that $N \cap H_{i}=f_{H_{i}}\left(S_{i}\right)$. Clearly $f_{H_{i}}\left(S_{i}\right) \subseteq N \cap H_{i}$. Let $y \in N \cap H_{i}$. This implies that $\bar{y}=1$, and thus $y \in S$. So $y=a_{1} a_{2} \cdots a_{n}$ where $a_{k} \in f_{H_{k}}\left(S_{k}\right)$, and $y a_{i}^{-1} \in H_{i} \cap \Pi_{k \neq i} H_{k}=1$. Hence $y=a_{i} \in f_{H_{i}}\left(S_{i}\right)$ and $N \cap H_{i}=f_{H_{i}}\left(S_{i}\right)$.

Next, we show that $N H_{i} \cap N H_{j}=N$. Clearly $N \subseteq N H_{i} \cap N H_{j}$. Let $x \in N H_{i} \cap N H_{j}$ where $i \neq j$. Then $x=n_{1} h_{i}=n_{2} h_{j}$ where $n_{1}, n_{2} \in N$, $h_{i} \in H_{i}$ and $h_{j} \in H_{j}$. This implies that $h_{i} h_{j}^{-1} \in N \cap H$. Therefore $\overline{h_{i} h_{j}^{-1}}=1$, and thus $h_{i} h_{j}^{-1} \in S$. Let $h_{i} h_{j}^{-1}=b_{1} b_{2} \cdots b_{n}$ where $b_{k} \in f_{H_{k}}\left(S_{k}\right)$. Then $h_{i} b_{i}^{-1} \in H_{i} \cap \Pi_{k \neq i} H_{k}=1$ and $h_{i}=b_{i} \in f_{H_{i}}\left(S_{i}\right) \subseteq N$. Hence $x \in N$ and $N H_{i} \cap N H_{j}=N$.

Lemma 3.2. Let $M$ and $K$ be finitely generated normal subgroups of $A_{r}$ and $A_{k}$ respectively where $M \cap \prod_{j \sim r} H_{r j}=1=K \cap \prod_{j \sim k} H_{k j}$ and $r \neq k$. Then for any $S_{r} \triangleleft_{f} M$ and $S_{k} \triangleleft_{f} K$, there exists $N \triangleleft_{f} G(T)$ such that $N \cap M=f_{M}\left(S_{r}\right)$, $N \cap K=f_{K}\left(S_{k}\right)$ and $N M \cap N K=N$.

Proof. By Lemma 3.1, there exists $N_{r} \triangleleft_{f} A_{r}$ such that $N_{r} \cap M=f_{M}\left(S_{r}\right)$ and $N_{r} \cap H_{r j}=f_{H_{r j}}\left(H_{r j}\right)=H_{r j}$ for all $j$ with $j \sim r$. Similarly, there exists $N_{k} \triangleleft_{f} A_{k}$ such that $N_{k} \cap K=f_{K}\left(S_{k}\right)$ and $N_{k} \cap H_{k j}=H_{k j}$ for all $j$ with $j \sim k$.

Let $N_{i}=\prod_{j \sim i} H_{i j}$ for $i \neq r, k$. Let $\phi_{i}$ be the natural epimorphism from $A_{i}$ onto $A_{i} / N_{i}$ for all $i \in V(T)=\{1,2, \ldots, n\}$. Then these epimorphisms can be extended to an epimorphism $\phi$ from $G(T)$ onto $\overline{G(T)}$ where $\overline{G(T)}=$ $A_{1} / N_{1} * A_{2} / N_{2} * \cdots * A_{n} / N_{n}$.

Since $\bar{G}$ is a free product and $r \neq k, \bar{M} \cap \bar{K}=1$. Since $\bar{G}$ is residually finite by [4, Theorem 2] and $\overline{M K}$ is finite, there exists $\bar{N} \triangleleft_{f} \bar{G}$ such that $\bar{N} \cap \overline{M K}=1$. Let $N$ be the preimage of $\bar{N}$. We shall show that $N$ is the required subgroup.

First we note that $N \cap M=N \cap A_{r} \cap M=N_{r} \cap M=f_{M}\left(S_{r}\right)$. Similarly, $N \cap K=f_{K}\left(S_{k}\right)$. So, it remains to show that $N M \cap N K=N$. Clearly $N \subseteq N M \cap N K$. Now let $y \in N M \cap N K$. Then $y=n_{1} m_{1}=n_{2} m_{2}$ where $n_{1}, n_{2} \in N, m_{1} \in M$ and $m_{2} \in K$. It is sufficient to show $m_{1} \in N$. Now 
$m_{1} m_{2}^{-1} \in N$ implies $\bar{m}_{1} \bar{m}_{2}^{-1} \in \bar{N} \cap \overline{M K}=1$. Since $\bar{M} \cap \bar{K}=1$, we have $\bar{m}_{1}=1$ and $m_{1} \in N$.

We shall need the following lemma of Kim [11, Theorem 2.3].

Lemma 3.3. Let $G=A_{H}^{*} B$ where $A, B$ are $H$-separable. Suppose for each $N_{H} \triangleleft_{f} H$, there exist $N_{A} \triangleleft_{f} A, N_{B} \triangleleft_{f} B$ such that $N_{A} \cap H=N_{B} \cap H \subseteq N_{H}$. Let $S$ be any subgroup of $B$. If $B$ is $S$-separable, then $G$ is $S$-separable.

Lemma 3.4. Let $M$ be a finitely generated subgroup of $A_{r}$. Then $G(T)$ is $M$-separable.

Proof. Let $V(T)=\{1,2, \ldots, n\}$. We shall use induction on $n$. The case $n=2$ follows from Lemma 3.1 and Lemma 3.3. Suppose $n \geq 3$. Note that the tree $T$ has a vertex of degree one, say $n$, which is joined to a unique vertex, say $n-1$. Let $T_{1}$ be the tree obtained by removing the vertex $n$ and the edge $n(n-1)$ from $T$. Then $G(T)=G\left(T_{1}\right)_{H}^{*} A_{n}$ where $H=H_{(n-1) n}=H_{n(n-1)}$. By induction, $G\left(T_{1}\right)$ is $H$-separable. By Lemma 3.2, for any $N_{H} \triangleleft_{f} H$, there exists $N_{1} \triangleleft_{f} G\left(T_{1}\right)$ such that $N_{1} \cap H=f_{H}\left(N_{H}\right)$. Since $A_{n}$ is subgroup separable, it is $H$-separable. By Lemma 3.1, there exists $N_{2} \triangleleft_{f} A_{n}$ such that $N_{2} \cap H=f_{H}\left(N_{H}\right)$.

Suppose $r=n$. Since $A_{n}$ is $M$-separable, by Lemma 3.3, G(T) is $M$ separable.

Suppose $r \neq n$. Then by induction, $G\left(T_{1}\right)$ is $M$-separable and thus by Lemma 3.3 again, $G(T)$ is $M$-separable.

We are now ready to prove Theorem 2.8 .

Proof of Theorem 2.8. We use induction on $n$. The case $n=2$ follows from Lemma 3.1 and Theorem 2.7. Suppose $n \geq 3$. As in Lemma 3.4, we may assume that $n$ is a vertex of degree one and is joined to a unique vertex, say $n-1$. Then $G(T)=G\left(T_{1}\right)_{H}^{*} A_{n}$ where $T_{1}$ is the tree obtained by removing the vertex $n$ and the edge $n(n-1)$ from $T$ and $H=H_{(n-1) n}=H_{n(n-1)}$. By Lemma 3.4, $G\left(T_{1}\right)$ is $H$-separable, and by Lemma 3.2, for any $N_{H} \triangleleft_{f} H$, there exists $N_{1} \triangleleft_{f} G\left(T_{1}\right)$ such that $N_{1} \cap H=f_{H}\left(N_{H}\right)$. Furthermore, $A_{n}$ is $H$-separable, and by Lemma 3.1, there exists $N_{2} \triangleleft_{f} A_{n}$ such that $N_{2} \cap H=f_{H}\left(N_{H}\right)$. Since $G\left(T_{1}\right)$ is $\pi_{c}$ by the induction hypothesis and $A_{n}$ is $\pi_{c}$, it follows from Theorem 2.7 that $G(T)$ is $\pi_{c}$.

\section{Proof of Lemma 2.9}

We shall need the following two lemmas from [25, Lemmas 4.1 and 4.2]

Lemma 4.1. Let $G=A_{H}^{*} B$ and $M, K$ be subgroups of $A, B$ respectively with $M \cap H=1=K \cap H$. Suppose for each $N_{H} \triangleleft_{f} H$ there exist $N_{A} \triangleleft_{f} A, N_{B} \triangleleft_{f} B$ such that $N_{A} \cap H=N_{B} \cap H \subseteq N_{H}$ and $N_{A} H \cap N_{A} M=N_{A}, N_{B} H \cap N_{B} K=N_{B}$. If $A$ is $H, M, H M, M H$-separable and $B$ is $H, K, H K, K H$-separable, then $G$ is $M K$-separable. 
Lemma 4.2. Let $G=A_{H}^{*} B$ and $M, K$ be subgroups of $A, B$ respectively with $M \cap H=1=K \cap H$. Suppose $A, B, M, K$ satisfy the hypothesis of Lemma 4.1. Then $G$ is $M * K$-separable.

Lemma 4.3. Let $G=A_{H}^{*} B$ and $M, K$ be subgroups of $B$. Suppose for each $N_{H} \triangleleft_{f} H$ there exist $N_{A} \triangleleft_{f} A, N_{B} \triangleleft_{f} B$ such that $N_{A} \cap H=N_{B} \cap H \subseteq N_{H}$. If $A$ is $H$-separable and $B$ is $H, M K$-separable, then $G$ is $M K$-separable.

Proof. Let $g \in G-M K$.

Case 1. $g \in B$. Since $B$ is $M K$-separable, there exists $P \triangleleft_{f} B$ such that $g \notin P M K$. Let $N_{H}=P \cap H$. By assumption, there exist $N_{A} \triangleleft_{f} A, N_{B} \triangleleft_{f} B$ such that $N_{A} \cap H=N_{B} \cap H \subseteq N_{H}$. Let $R_{A}=N_{A}, R_{B}=N_{B} \cap P$. Then $R_{A} \cap H=R_{B} \cap H$. Let $\bar{G}=A / R_{A} \frac{*}{H} B / R_{B}$ where $\bar{H}=H R_{A} / R_{A}=H R_{B} / R_{B}$. Clearly, $\bar{G}$ is a homomorphic image of $G$, and $\bar{g} \notin \overline{M K}$. Since $\bar{G}$ is residually finite by [4, Theorem 2] and $\overline{M K}$ is finite, there exists $\bar{N} \triangleleft_{f} \bar{G}$ such that $\bar{g} \notin \overline{N M K}$. Let $N$ be the preimage of $\bar{N}$. Then $g \notin N M K$.

Case 2. $g \in A-H$ or $g \notin A \cup B$. We will only consider the case $g=$ $a_{1} b_{1} a_{2} b_{2} \cdots a_{n} b_{n}$ where $a_{i} \in A-H, b_{i} \in B-H, i=1,2, \ldots, n$. The other cases are similar. Since $A$ is $H$-separable, $B$ is $H$-separable, there exist $M_{A} \triangleleft_{f} A$, $M_{B} \triangleleft_{f} B$ such that $a_{i} \notin M_{A} H, b_{i} \notin M_{B} H, i=1,2, \ldots, n$. Let $N_{H}=M_{A} \cap M_{B}$. By assumption, there exist $N_{A} \triangleleft_{f} A, N_{B} \triangleleft_{f} B$ such that $N_{A} \cap H=N_{B} \cap H \subseteq$ $N_{H}$. Let $R_{A}=N_{A} \cap M_{A}, R_{B}=N_{B} \cap M_{B}$. Then $R_{A} \cap H=R_{B} \cap H$. Let $\bar{G}=A / R_{A} \frac{*}{H} B / R_{B}$ where $\bar{H}=H R_{A} / R_{A}=H R_{B} / R_{B}$. Clearly, $\|\bar{g}\|=\|g\|$. So $\bar{g} \notin \overline{M K}$. Furthermore, $\overline{M K}$ is finite. We can now proceed as in Case 1 to complete the proof.

Lemma 4.4. Let $M$ and $K$ be finitely generated normal subgroups of $A_{r}$ and $A_{k}$ respectively where $M \cap \prod_{j \sim r} H_{r j}=1=K \cap \prod_{j \sim k} H_{k j}$ (here we allow $r=k)$. Then $G(T)$ is $M K$-separable.

Proof. Let $V(T)=\{1,2, \ldots, n\}$. We shall use induction on $n$. The case $n=2$ follows from Lemma 3.1, Lemma 4.1 and Lemma 4.3. Suppose $n \geq 3$. As in Lemma 3.4, we may assume that $n$ is a vertex of degree one and is joined to a unique vertex, say $n-1$. Then $G(T)=G\left(T_{1}\right)_{H}^{*} A_{n}$ where $T_{1}$ is the tree obtained by removing the vertex $n$ and the edge $n(n-1)$ from $T$ and $H=H_{(n-1) n}=H_{n(n-1)}$.

Case 1. $M \leq G\left(T_{1}\right)$ and $K \leq A_{n}$. By the induction, $G\left(T_{1}\right)$ is $M H, H M$ separable. By Lemma 3.4, $G\left(T_{1}\right)$ is $M, H$-separable. By Lemma 3.2, for any $S_{H} \triangleleft_{f} H, S_{M} \triangleleft_{f} M$ and $S_{K} \triangleleft_{f} K$, there exists $N_{1} \triangleleft_{f} G\left(T_{1}\right)$ such that $N_{1} \cap M=$ $f_{M}\left(S_{M}\right), N_{1} \cap H=f_{H}\left(S_{H}\right)$ and $N_{1} M \cap N_{1} H=N_{1}$. By Lemma 3.1, there exists $N_{2} \triangleleft_{f} A_{n}$ such that $N_{2} \cap K=f_{K}\left(S_{K}\right), N_{2} \cap H=f_{H}\left(S_{H}\right)$ and $N_{2} K \cap N_{2} H=N_{2}$.

Since $A_{n}$ is subgroup separable, it is $H, K, H K, K H$-separable. Hence by Lemma 4.1, $G(T)$ is $M K$-separable.

Case 2. $K \leq G\left(T_{1}\right)$ and $M \leq A_{n}$. The argument is similar to Case 1 .

Case 3. $K, M \leq G\left(T_{1}\right)$. By induction, $G\left(T_{1}\right)$ is $M K$-separable. By Lemma 3.4, $G\left(T_{1}\right)$ is $H$-separable, and by Lemma 3.2, for any $S_{H} \triangleleft_{f} H$ there exists 
$N_{1} \triangleleft_{f} G\left(T_{1}\right)$ such that $N_{1} \cap H=f_{H}\left(S_{H}\right)$. Furthermore, $A_{n}$ is $H$-separable, and by Lemma 3.1, for any $S_{H} \triangleleft_{f} H$ there exists $N_{2} \triangleleft_{f} A_{n}$ such that $N_{2} \cap H=$ $f_{H}\left(S_{H}\right)$. Hence by Lemma $4.3, G(T)$ is $M K$-separable.

Case 4. $K, M \leq A_{n}$. The argument is similar to Case 3 .

We are now ready to prove Lemma 2.9.

Proof of Lemma 2.9. Let $V(T)=\{1,2, \ldots, n\}$. We shall use induction on $n$. The case $n=2$ follows from Lemma 3.1 and Lemma 4.2. Suppose $n \geq 3$. As in Lemma 3.4, we may assume that $n$ is a vertex of degree one and is joined to a unique vertex, say $n-1$. Then $G(T)=G\left(T_{1}\right)_{H}^{*} A_{n}$ where $T_{1}$ is the tree obtained by removing the vertex $n$ and the edge $n(n-1)$ from $T$ and $H=H_{(n-1) n}=H_{n(n-1)}$.

Case 1. $M \leq G\left(T_{1}\right)$ and $K \leq A_{n}$. By Lemma 4.4, $G\left(T_{1}\right)$ is $M H$-separable and $H M$-separable. By Lemma 3.4, $G\left(T_{1}\right)$ is $M, H$-separable. By Lemma 3.1 and Lemma 3.2, $G\left(T_{1}\right)$ and $A_{n}$ satisfy the hypothesis of Lemma 4.2. Hence $G(T)$ is $M * K$-separable.

Case 2. $K \leq G\left(T_{1}\right)$ and $M \leq A_{n}$. The argument is similar to Case 1 .

Case 3. $K, M \leq G\left(T_{1}\right)$. By induction, $G\left(T_{1}\right)$ is $M * K$-separable. By Lemma 3.4, $G\left(T_{1}\right)$ is $H$-separable. It then follows from Lemma 3.1, Lemma 3.2 and Lemma 3.3 that $G(T)$ is $M * K$-separable.

\section{Proof of Lemma 2.10}

Lemma 5.1. Let $G=A_{H}^{*} B$ and $M, K$ be subgroups of $A, B$ respectively with $M \cap H=1=K \cap H$. Let $S \triangleleft_{f}(M * K)$ and suppose there exist $N_{A} \triangleleft_{f} A, N_{B} \triangleleft_{f} B$ such that $N_{A} \cap H=H=N_{B} \cap H$ and $N_{A} \cap M=S \cap M, N_{B} \cap K=S \cap K$. Then there exists $N \triangleleft_{f} G$ such that $N \cap(M * K)=S$.

Proof. Let $\bar{G}=A / N_{A} * B / N_{B}$. Since $N_{A} \cap H=H=N_{B} \cap H$, the natural epimorphisms from $A$ onto $A / N_{A}$ and from $B$ onto $B / N_{B}$ can be extended to an epimorphism $\psi$ from $G$ onto $\bar{G}$.

First we show that $\operatorname{Ker} \psi \cap(M * K) \subseteq S$. Let $g$ be any element with the smallest length such that $g \in \operatorname{Ker} \psi \cap(\bar{M} * K)$ but $g \notin S$. Without loss of generality we may assume $g=m_{1} k_{1} \cdots m_{n} k_{n}$ where $m_{i} \in M, k_{i} \in K$. Then $\bar{g}=\overline{m_{1} k_{1} \cdots m_{n} k_{n}}=1$. Hence $\bar{m}_{i}=1$ or $\bar{k}_{i}=1$ for some $i$. Suppose $\bar{m}_{i}=1$ (the case $\bar{k}_{i}=1$ is similar). Then $m_{i} \in N_{A}$ for $\bar{M}=M N_{A} / N_{A}$. Hence $m_{i} \in N_{A} \cap M=S \cap M \subseteq S$. Now

$g=\left(m_{1} k_{1} \cdots m_{i-1} k_{i-1}\right) m_{i}\left(m_{1} k_{1} \cdots m_{i-1} k_{i-1}\right)^{-1}\left(m_{1} k_{1} \cdots m_{i-1} k_{i-1}\right) k_{i} \cdots m_{n} k_{n}$.

But $\left(m_{1} k_{1} \cdots m_{i-1} k_{i-1}\right) m_{i}\left(m_{1} k_{1} \cdots m_{i-1} k_{i-1}\right)^{-1} \in S$ since $m_{i} \in S \triangleleft_{f}(M * K)$. This implies that $g_{1}=\left(m_{1} k_{1} \cdots m_{i-1} k_{i-1}\right) k_{i} \cdots m_{n} k_{n} \notin S$. Furthermore $\overline{g_{1}}=$ 1 for $\bar{m}_{i}=1$. Therefore $g_{1} \in \operatorname{Ker} \psi \cap(M * K)$, but $\left\|g_{1}\right\|<\|g\|$, a contradiction. Thus Ker $\psi \cap(M * K) \subseteq S$.

Next we show that $\bar{S} \cap \bar{M}=1$ and $\bar{S} \cap \bar{K}=1$. Let $\bar{y} \in \bar{S} \cap \bar{M}$. Then $\bar{y}=\bar{s}=\bar{m}$ where $s \in S$ and $m \in M$. So $\bar{s} \overline{m^{-1}}=1$, and this implies that 
$s m^{-1} \in \operatorname{Ker} \psi \cap(M * K) \subseteq S$. Therefore $m \in S \cap M \subseteq N_{A}$. This implies that $\bar{m}=1$, and thus $\bar{y}=1$. Hence $\bar{S} \cap \bar{M}=1$. Similarly, $\bar{S} \cap \bar{K}=1$.

Now $\bar{G}=A / N_{A} * B / N_{B}=A / N_{A} \frac{*}{M}(\bar{M} * \bar{K}) \frac{*}{K} B / N_{B}$. Since $\bar{S} \cap \bar{M}=1$, $\overline{\bar{M}}=\overline{M S} / \bar{S} \simeq \bar{M} /(\bar{S} \cap \bar{M}) \simeq \bar{M}$. Similarly, $\bar{S} \cap \bar{K}=1$ implies that $\overline{\bar{K}}=$ $\overline{K S} / \bar{S} \simeq \bar{K} /(\bar{S} \cap \bar{K}) \simeq \bar{K}$. So, we can form $\overline{\bar{G}}=A / N_{A} \frac{*}{\bar{M}}((\bar{M} * \bar{K}) / \bar{S}) \frac{*}{\bar{K}} B / N_{B}$. Clearly, $\overline{\bar{G}}$ is a homomorphic image of $\bar{G}$, and thus $\overline{\bar{G}}$ is a homomorphic image of $G$.

Since $(\bar{M} * \bar{K}) / \bar{S}$ is finite and $\overline{\bar{G}}$ is residually finite by [4, Theorem 2], there exists $\overline{\bar{N}} \triangleleft_{f} \overline{\bar{G}}$ such that $\overline{\bar{N}} \cap((\bar{M} * \bar{K}) / \bar{S})=1$. Let $N$ be the preimage of $\overline{\bar{N}}$ in $G$. We shall show that $N \cap(M * K)=S$. Clearly $S \subseteq N \cap(M * K)$. Now let $g_{2} \in N \cap(M * K)$. Since $\overline{\overline{g_{2}}}=1$, we have $\overline{g_{2}} \in \bar{S}$. Let $\overline{g_{2}}=\bar{t}$ where $t \in S$. Then $\overline{g_{2}} \overline{t^{-1}}=1$, and this implies that $g_{2} t^{-1} \in \operatorname{Ker} \psi \cap(M * K) \subseteq S$. Hence $g_{2} \in S$ and $N \cap(M * K)=S$.

We are now ready to prove Lemma 2.10.

Proof of Lemma 2.10. Let $S \triangleleft_{f}(M * K)$ be given. Let $S_{M}=f_{M * K}(S) \cap M$ and $S_{K}=f_{M * K}(S) \cap K$. Then $S_{M}$ and $S_{K}$ are characteristic subgroups of $M$ and $K$, respectively.

Since $T$ is a tree, there exists a unique path from the vertex $r$ to the vertex $k$. Let $i_{0} j_{0}$ be an edge on this path. Let $T_{1}$ and $T_{2}$ be the two disjoint trees obtained by removing the edge $i_{0} j_{0}$ from $T$. Then $G(T)=G\left(T_{1}\right)_{H}^{*} G\left(T_{2}\right)$ where $H=H_{i_{0} j_{0}}=H_{j_{0} i_{0}}$ and $A_{r} \subseteq G\left(T_{1}\right)$ and $A_{k} \subseteq G\left(T_{2}\right)$. For all $j$ with $j \sim r$, let $S_{r j}=H_{r j}$, and for all $j$ with $j \sim k$, let $S_{k j}=H_{k j}$.

By Lemma 3.1, there exists $N_{r} \triangleleft_{f} A_{r}$ such that $N_{r} \cap H_{r j}=f_{H_{r j}}\left(S_{r j}\right)=H_{r j}$ and $N_{r} \cap M=f_{M}\left(S_{M}\right)=S_{M}$. Let $\psi_{1}$ be the epimorphism from $G\left(T_{1}\right)$ onto $A_{r} / N_{r}$ where $\psi_{1}(a)=1, \forall a \in A_{i} \subseteq G\left(T_{1}\right), i \neq r$ and $\psi_{1}(a)=a N_{r}, \forall a \in$ $A_{r}$. Let $N_{1}=K e r \psi_{1}$. Then $N_{1} \triangleleft_{f} G\left(T_{1}\right), N_{1} \cap M=f_{M}\left(S_{M}\right)=S_{M}$ and $N_{1} \cap H=H$. Similarly there is a $N_{2} \triangleleft_{f} G\left(T_{2}\right)$ with $N_{2} \cap K=f_{K}\left(S_{K}\right)=S_{K}$ and $N_{2} \cap H=H$. Therefore by Lemma 5.1, there exists $N \triangleleft_{f} G(T)$ such that $N \cap(M * K)=f_{M * K}(S)$.

\section{References}

[1] R. B. J. T. Allenby, Polygonal products of polycyclic by finite groups, Bull. Austral. Math. Soc. 54 (1996), no. 3, 369-372.

[2] R. B. J. T. Allenby, G. Kim, and C. Y. Tang, Residual finiteness of outer automorphism groups of certain pinched 1-relator groups, J. Algebra 246 (2001), no. 2, 849-858.

[3] — Residual finiteness of outer automorphism groups of finitely generated nontriangle Fuchsian groups, Internat. J. Algebra Comput. 15 (2005), 59-72.

[4] G. Baumslag, On the residual finiteness of generalised free products of nilpotent groups, Trans. Amer. Math. Soc. 106 (1963), 193-209.

[5] A. M. Brunner, M. L. Frame, Y. W. Lee, and N. J. Wielenberg, Classifying torsion-free subgroups of the Picard group, Trans. Amer. Math. Soc. 282 (1984), no. 1, 205-235.

[6] Y. D. Chai, Y. Choi, G. Kim, and C. Y. Tang, Outer automorphism groups of certain tree products of abelian groups, Bull. Austral. Math. Soc. 77 (2008), no. 1, 9-20. 
[7] B. Fine, J. Howie, and G. Rosenberger, One-relator quotients and free products of cyclics, Proc. Amer. Math. Soc. 102 (1988), no. 2, 249-254.

[8] E. K. Grossman, On the residual finiteness of certain mapping class groups, J. London Math. Soc. 2 (1974), 160-164.

[9] M. Hall Jr., Coset representations in free groups, Trans. Amer. Math. Soc. 67 (1949), 421-432.

[10] A. Karrass, A. Pietrowski, and D. Solitar, The subgroups of polygonal products of groups, (unpublished manuscript).

[11] G. Kim, On polygonal products of finitely generated abelian groups, Bull. Austral. Math. Soc. 45 (1992), no. 3, 453-462.

[12] _ Cyclic subgroup separability of generalized free products, Canad. Math. Bull. 36 (1993), no. 3, 296-302.

[13] _ Cyclic subgroup separability of HNN extensions, Bull. Korean Math. Soc. 30 (1993), no. 2, 285-293.

[14] - Outer automorphism groups of certain polygonal products of groups, Bull. Korean Math. Soc. 45 (2008), no. 1, 45-52.

[15] G. Kim and C. Y. Tang, A criterion for the conjugacy separability of amalgamated free products of conjugacy separable groups, J. Algebra 184 (1996), no. 3, 1052-1072.

[16] Conjugacy separability of generalized free products of finite extension of residually nilpotent groups, in "Group Theory (Proc.'96 Intl. Symposium)", 10-24, SpringerVerlag, Berlin, 1998.

[17] — Cyclic subgroup separability of HNN-extensions with cyclic associated subgroups, Canad. Math. Bull. 42 (1999), no. 3, 335-343.

[18] - Outer automorphism groups of polygonal products of certain conjugacy separable groups, J. Korean Math. Soc. 45 (2008), no. 6, 1741-1752.

[19] A. I. Mal'cev, On homomorphisms onto finite groups, Ivanov. Gos Ped. Inst. Ucen. Zap. 18 (1958), 49-60.

[20] V. Metaftsis and E. Raptis, Subgroup separability of HNN extensions with abelian base groups, J. Algebra 245 (2001), no. 1, 42-49.

[21] E. Raptis, O. Talelli, and D. Varsos, On the conjugacy separability of certain graphs of groups, J. Algebra 199 (1998), no. 1, 327-336.

[22] P. Stebe, Residual finiteness of a class of knot groups, Comm. Pure Appl. Math. 21 (1968), 563-583.

[23] C. Y. Tang, Conjugacy separability of generalized free products of certain conjugacy separable groups, Canad. Math. Bull. 38 (1995), no. 1, 120-127.

[24] _ Conjugacy separability of generalized free products of surface groups, J. Pure Appl. Algebra 120 (1997), no. 2, 187-194.

[25] K. B. Wong and P. C. Wong, Polygonal products of residually finite groups, Bull. Korean Math. Soc. 44 (2007), no. 1, 61-71.

[26] _ Conjugacy separability and outer automorphism groups of certain HNN extensions, J. Algebra 334 (2011), 74-83.

[27] _ Residual finiteness, subgroup separability and Conjugacy separability of certain HNN extensions, Math. Slovaca 62 (2012), no. 5, 875-884.

[28] P. C. Wong and K. B. Wong, The cyclic subgroup separability of certain HNN extensions, Bull. Malays. Math. Sci. Soc. (2) 29 (2006), no. 2, 111-117.

[29] _ Residual finiteness of outer automorphism groups of certain tree products, J. Group Theory 10 (2007), no. 3, 389-400.

[30] - Subgroup separability and conjugacy separability of certain HNN extensions, Bull. Malays. Math. Sci. Soc. (2) 31 (2008), no. 1, 25-33.

[31] W. Zhou and G. Kim, Class-preserving automorphisms and inner automorphisms of certain tree products of groups, J. Algebra 341 (2011), 198-208. 
[32] — Class-preserving automorphisms of generalized free products amalgamating a cyclic normal subgroup, Bull. Korean Math. Soc. 49 (2012), no. 5, 949-959.

KOK Bin WONG

Institute of Mathematical Sciences

UNIVERSITY OF MALAYA

50603 Kuala Lumpur, Malaysia

E-mail address: kbwong@um.edu.my

Peng Choon Wong

Institute of Mathematical SCIENCES

UNIVERSiTY OF MALAYA

50603 Kuala Lumpur, Malaysia

E-mail address: wongpc@um.edu.my 